\title{
Chronic Alcohol Causes Alteration of Lipidome Profiling in Brain
}

\author{
Wang, Liang
}

2019-10-01

Wang , L , Li , M , Bu , Q , Li , H , Xu , W , Liu , C , Gu, H, Zhang , J , Wan , X , Zhao , Y \&

Cen , X 2019 , ' Chronic Alcohol Causes Alteration of Lipidome Profiling in Brain ' ,

Toxicology Letters , vol. 313 , pp. 19-29 . https://doi.org/10.1016/j.toxlet.2019.05.010

http://hdl.handle.net/10138/314746

https://doi.org/10.1016/j.toxlet.2019.05.010

cc_by_nc_nd

acceptedVersion

Downloaded from Helda, University of Helsinki institutional repository.

This is an electronic reprint of the original article.

This reprint may differ from the original in pagination and typographic detail.

Please cite the original version. 


\section{Accepted Manuscript}

Title: Chronic Alcohol Causes Alteration of Lipidome Profiling in Brain

Authors: Liang Wang, Menglu Li, Qian Bu, Hongchun Li, Wei Xu, Chunqi Liu, Hui Gu, Jiamei Zhang, Xuemei Wan, Yinglan Zhao, Xiaobo Cen

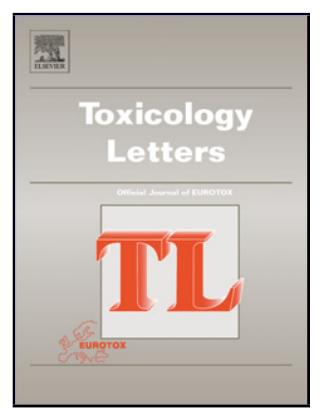

PII: S0378-4274(19)30143-2

DOI:

Reference: https://doi.org/10.1016/j.toxlet.2019.05.010

To appear in: TOXLET 10485

Received date: $\quad 2$ March 2019

Revised date: $\quad 6$ May 2019

Accepted date: $\quad 9$ May 2019

Please cite this article as: Wang L, Li M, Bu Q, Li H, Xu W, Liu C, Gu H, Zhang J, Wan X, Zhao Y, Cen X, Chronic Alcohol Causes Alteration of Lipidome Profiling in Brain, Toxicology Letters (2019), https://doi.org/10.1016/j.toxlet.2019.05.010

This is a PDF file of an unedited manuscript that has been accepted for publication. As a service to our customers we are providing this early version of the manuscript. The manuscript will undergo copyediting, typesetting, and review of the resulting proof before it is published in its final form. Please note that during the production process errors may be discovered which could affect the content, and all legal disclaimers that apply to the journal pertain. 


\title{
Chronic Alcohol Causes Alteration of Lipidome Profiling in Brain
}

\author{
Liang Wang ${ }^{1,3 \#}$, Menglu Li ${ }^{1 \#}$, Qian $\mathrm{Bu}^{1,2}$, Hongchun $\mathrm{Li}^{1}$, Wei Xu${ }^{1}$, Chunqi $\mathrm{Liu}^{1}$, Hui Gu${ }^{1}$, \\ Jiamei Zhang ${ }^{1}$, Xuemei Wan ${ }^{1}$, Yinglan Zhao ${ }^{1}$, Xiaobo Cen $^{1 *}$
}

${ }^{1}$ National Chengdu Center for Safety Evaluation of Drugs, State Key Laboratory of Biotherapy and Cancer Center, West China Hospital, Sichuan University, and Collaborative Innovation Center for Biotherapy, Chengdu 610041, China.

${ }^{2}$ Healthy Food Evaluation Research Center, Department of Food Science and Technology, College of Light Industry, Textile and Food Engineering, Sichuan University, Chengdu 610065, China.

${ }^{3}$ Institute of Biotechnology, University of Helsinki, Finland

* To whom correspondence should be addressed to Xiaobo Cen,

Tel: +86-28-85178767; E-mail: xbcen@scu.edu.cn

\# Liang Wang and Menglu Li contribute equally. 


\section{Highlights}

1. Chronic alcohol exposure profoundly modifies lipidome profiling in PFC and striatum.

2. Three lipid classes, GP, GL and FA, take the most proportion of those modified lipidome.

3. The majority of altered lipids are synthesized in ER.

4. ER stress may account for the neurotoxicity of chronic alcohol caused disorders.

\section{ABSTRACT}

Much efforts have been tried to clarify the molecular mechanism of alcohol-induced brain damage from the perspective of genome and protein; however, the effect of chronic alcohol exposure on global lipid profiling of brain is unclear. In the present study, by using QTOF/MS-based lipidomics approach, we investigated the comprehensive lipidome profiling of brain from the rats orally administrated with alcohol daily, continuously for one year. Through systematically analysis of all lipids in prefrontal cortex (PFC) and striatum region, we found that long-term alcohol exposure profoundly modified brain lipidome profiling. Notably, three kinds of lipid classes, glycerophospholipid (GP), glycerolipid (GL) and fatty acyls (FA), were significantly increased in these two brain regions. Interestingly, most of the modified lipids were involved in synthetic pathways of endoplasmic reticulum (ER), which may result in ER stress-related metabolic disruption. Moreover, alcohol-modified lipid species displayed long length of carbon chain with high degree of unsaturation. Taken together, our results firstly present that chronic alcohol exposure markedly modifies brain lipidomic profiling, which may activate ER stress and eventually result in neurotoxicity. These findings provide a new insight into the mechanism of alcohol-related brain damage.

\section{Abbreviations:}


Q-TOF/MS, quadrupole time of flight mass spectrometry; PFC, prefrontal cortex; ER, endoplasmic reticulum; AUD, alcohol use disorder; CNS, central nervous system; AD, Alzheimer's disease; MTBE, methyl-tert-butyl ether; MS, mass spectrometry; PCA, principal component analysis; OPLS-DA, orthogonal partial least squares discriminant analysis; VIP, variable importance; TIC, representative total ion current; CDP-DAG, cytidine diphosphatediacylglycerol; ASMase, acid sphingomyelinase; DG, diacylglycerol; PS, phosphatidylserine; PC, phosphatidylcholine; PI, phosphatidylinositol; MG, mono-acylglycerol; PE, phosphatidylethanolamine; FA, fatty acids; PG, phosphatidylglycerol; PA, phosphatidic acid; Sulfo, 2-hydroxy N-acyl sulfatide ; LPC, lysophosphatidylcholine; TG, triacylglycerol; Cer, ceramide; LacCer, lactosylceramide; GM3, ganglioside GM3; D3(OH3), 1,23,25trihydroxyvitamin D3; LPE, lysophosphatidylethanolamine; AA, arachidonic acid; CE, cholesteryl ester; OLGly, N-oleoyl glycine; VC, vaccenyl carnitine; AGMI, archaetidylglycerol-(glycosyl)-myo-inositol; E3-3GI, estriol-3-glucuronide; Cho, cholesterol; COQ9, Coenzyme Q9; COQ10, Coenzyme Q10; SP, sphingolipids; GL, glycerolipids; ST, sterol Lipids; GP, glycerophospholipids; FA, fatty Acyls.

\section{Key words: Alcohol abuse; Lipidome profiling; ER stress}




\section{Introduction}

Alcohol-related disorders cause about 3-8\% of all globe deaths and 4-6\% of global disabilityadjusted life years (Deak, Miller, and Gizer 2019; Kranzler and Soyka 2018). Aberrant genetic variation and synaptic functions induced by long-term alcohol exposure involve in the pathogenesis of many categories of alcohol use disorder (AUD), such as neuropsychiatric impairment and cardiovascular diseases (Mayfield et al. 2016; Parry, Patra, and Rehm 2011; Rehm et al. 2010; Rehm and Ph 2012). Alcohol abuse produces neuroadaptations in specific brain circuits that are linked with behavioral indices, including escalating alcohol consumption, tolerance, dependence, and propensity to relapse after a period of abstinence (Becker and Dorit Ron Guest 2014; Erickson et al. 2018). Especially, chronic alcohol drinking arouses permanent structural and functional damage to brain accompanying with severe cognitive and motor impairments (Harper 2009; He et al. 2007).

Lipids hemostasis of the brain plays an important role in the metabolism and function of central nervous system (CNS). Oxidation of ethanol (alcohol) generates hydroxyethyl or superoxide radical, which react with and damage complex cellular molecules, such as lipids, proteins and DNA (Domnina et al. 2015; Pamplona et al. 2004; Schweikl et al. 2016). These processes play key roles in the mechanism of alcohol-promoted tissue injury (Cederbaum and $\mathrm{Lu}$ 2009). Through directly interacting with side chain of polyunsaturated fatty acids or increase the expression level of lipoxidation and lipid peroxides, the oxidative stress has capability to disrupt the stability of lipids and results in structural membrane damage (Cederbaum and Lu 2009; Dey and Cederbaum 2006). Because lipids account for the major mass of brain, except for as the building blocks for all membrane structures, they also serve as repositories of chemical energy, as well as play key roles in cell signaling and transduction pathways (Holthuis and Menon 2014; Nutabi et al. 2017). Therefore, changes in lipid 
structures, composition, distribution, profiles or lipid raft micro-domains are considered to associate with altered neuronal function, synaptic transmission and neurotransmitter signaling (Allen, Halverson-tamboli, and Rasenick 2007; Fabelo et al. 2011; Ferrer 2009; De La Monte and Kril 2014; Ramírez et al. 2009). In the human frontal cortex of late stages of Alzheimer's disease (AD) and Parkinson's disease, alteration of lipid composition and profiles involves in the neuronal dysfunction and cognitive deterioration (Fabelo et al. 2011; Marin et al. 2017). Furthermore, accumulation of adducts formed from toxic metabolites of ethanol, acetaldehyde or fatty acid ethyl esters, disrupts lipid membrane structure and mitochondrial function, inducing neuronal damage in brain tissue (Cui et al. 2019; Inoue et al. 2018; Nakamura et al. 2003; Zahr, Kaufman, and Harper 2011). Our recent study revealed that long-term alcohol exposure significantly modifies the serum lipid profile in rats, particularly the metabolic pathways involved in glycerophospholipid, sphingolipid and glycerolipids (Li et al. 2018). Though many efforts have tried to explore the pathological features of alcohol-related brain damage, few studies have systematically investigated the global impact of alcohol on brain lipidome profiling, especially long-term alcohol exposure.

Systemic analysis of lipids and their metabolites in complex mixtures can be achieved using the novel analytical approaches, especially liquid chromatography and mass spectrometry (Shevchenko and Simons 2010; Wenk 2005). Growing lipidomics-based studies have revealed that the dysregulation of some specific lipids are associated with the pathogenesis of many disease, such as diabetes, Alzheimer's disease, hypertension and cancer (Gao et al. 2018; Kulkarni et al. 2017; Links 2011; Yan et al. 2018). In this study, using Q-TOF/MS based lipidomic approach, we show that alcohol exposure markedly remodels the lipidome of both prefrontal cortex and striatum of the rats, which may underlie alcohol-related neurotoxicity and neuroplasticity. 


\section{Materials and Methods}

\subsection{Reagents}

Absolute alcohol was bought from Taineng Co., Ltd (Chengdu, China). Isopropanol, acetonitrile, ammonium acetate and formic acid were purchased from Sigma-Aldrich (USA). All of organic solutions used in this study were of analytic pure.

\subsection{Animals}

Wistar rats (5 6 weeks old, 130 180 g weights) were purchased from Vital River Laboratory Animal Technology Co., Ltd. (Beijing, China) and allowed to acclimated for 7 days before alcohol exposure. All rats were maintained in the animal facility under a standard 12-h light/12-h dark cycle with food and water ad libitum. All experimental procedures and use of the animals were in accordance with the guidelines established by the Association for Assessment and Accreditation of Laboratory Animal Care and the Institutional Animal Care and Use Committee of Sichuan University.

\subsection{Alcohol exposure}

Rats were randomly divided into water-treated $(n=10)$ and alcohol-treated $(n=10)$ groups. The alcohol-treated rats were administrated intragastrically with $10 \mathrm{ml} / \mathrm{kg}$ of alcohol $(12 \%, \mathrm{v} / \mathrm{v})$ daily throughout one year without interruption, and the water-treated rats were administrated intragastrically with $10 \mathrm{ml} / \mathrm{kg}$ of distilled water daily.

\subsection{Sample preparation}

Rat were anesthetized with $60 \mathrm{mg} / \mathrm{kg}$ pentobarbital sodium intraperitoneally at the end of administration. After quickly excising the brain, prefrontal cortex and striatum were immediately dissected according to the brain atlas (Paxinos and Franklin, 2004) and frozen in liquid nitrogen before storage at $-80{ }^{\circ} \mathrm{C}$.

\subsection{Lipid extraction}


Total lipids were extracted with methyl-tert-butyl ether (MTBE) (Matyash et al. 2008). Simply, pre-cold methanol $(150 \mu \mathrm{l})$ and MTBE $(450 \mu \mathrm{l})$ were sequentially added to brain tissues (25-30 mg). After incubating for $10 \mathrm{~min}$ at room temperature, the brain tissues were followed by homogenization using a bead based homogenizer (Precellys Evolution, Bertin technologies, Montigny le Bretonneux, France) at $6500 \mathrm{rpm}$ in three cycles for $15 \mathrm{~s}$ with a 20 -s intervals. Then, $300 \mu 125 \%$ methanol diluted in sterile MiliQ water were added to homogenized mixture and phase separation was formed. After mixing vigorously and centrifugation at $14000 \mathrm{~g}$ for $10 \mathrm{~min}$, the upper organic phase was carefully collected. The extracted lipids were subjected to evaporation under a gentle stream of nitrogen at room temperature and then stored at $-80{ }^{\circ} \mathrm{C}$ until use.

\subsection{Mass spectrometry analysis of lipid metabolites}

The extracted lipids from PFC and striatum were separated by an ACQUITY Ultra Performance LC mass spectrometry system (Milford MA, US) with an ACQUITY UPLC HSS T3 column $(1.8 \mu \mathrm{m}, 2.1 \times 100 \mathrm{~mm}$; Milford MA, US $)$ and measured by electro-spray ionization (ESI)-Q-TOF (quadrupole-time-of-flight) Premier mass spectrometer (Milford MA, US). The dried lipid samples were re-dissolved in acetonitrile/isopropanol (v/v, 7:3). The injection volume was fixed at $3 \mu \mathrm{l}$, The T3 column was maintained at $55^{\circ} \mathrm{C}$. Mobile phase A consisted of acetonitrile/water $=4 / 6(10 \mathrm{mmol}$ ammonium acetate $)$. Mobile phase $\mathrm{B}$ consisted of acetonitrile/isopropanol $=1 / 9(10 \mathrm{mmol}$ ammonium acetate $)$. The flow rate of the mobile phase was maintained at $0.4 \mathrm{ml} / \mathrm{min}$. A linear gradient was used as follows: $40-70 \% \mathrm{~B}$ at $0-3$ $\min , 70-95 \% \mathrm{~B}$ at $3-14 \mathrm{~min}, 95 \% \mathrm{~B}$ at $14-15.5 \mathrm{~min}$. The column was re-equilibrated for 3.5 min, setting a total run time of $20 \mathrm{~min}$ (Castro-perez et al. 2010).

The mass spectrometry (MS) was separately operated in the positive and negative modes. The scan range of the instrument was set at a mass-to-charge ratio $(\mathrm{m} / \mathrm{z})$ of $50-1200$. The capillary and cone voltage in the positive ion mode were set at $3.0 \mathrm{kV}$ and $40 \mathrm{~V}$, respectively. In the 
negative ion detection mode, the cone voltage was changed to $-40 \mathrm{~V}$, while set the same capillary voltage with the positive ion mode. The desolvation gas was set to $600 \mathrm{l} / \mathrm{h}$ at a temperature of $300{ }^{\circ} \mathrm{C}$; the cone gas was adjusted to $50 \mathrm{l} / \mathrm{h}$ and the source temperature was $120{ }^{\circ} \mathrm{C}$ (Want et al. 2013). A lock spray is necessary for accuracy. Leucine-encephalin (4 $\mathrm{ng} / \mathrm{ml})$ was used for the positive and negative ion modes $\left([\mathrm{M}+\mathrm{H}]^{+}=556.2771\right.$ and $[\mathrm{M}+\mathrm{H}]^{-}=$ 554.2615). The data were acquired in the continuous mode using MassLynx (Milford MA, US).

\subsection{Data processing and analysis}

UPLC-ESI-TOFMS data was processed by the Progenesis QI software (Newcastle, UK). The analysis process was sequentially performed, from alignment, peak picking to identification of lipids. Metabolites were identified by mainly referring to the Lipid Maps Database (www.lipidmaps.org) and the Human Metabolome Database (http://www.hmdb.ca/). Data sheets from Progenesis QI software were obtained and absolute intensities of all identified compounds were recalculated to relative abundances of lipid molecules. Pareto scaling was used for final statistical models. The data were processed by unsupervised principal component analysis (PCA) to obtain group clusters. (OPLS-DA is often used in lieu of PLSDA to disentangle group predictive and group-unrelated variation in the measured data. In doing so, OPLS-DA constructs more parsimonious and easily interpretable models compared to PLS-DA (Worley and Powers 2013, 2016). Lipid molecules with the highest impact on the group clustering were identified in the variable importance (VIP)-plots (VIP $\geq 1$ ). Besides, unpaired Student's t-test $(p<0.05)$ to the chemical shifts was also used to assess the significance of each metabolite. The metabolites in two groups which showed both VIP $\geq 1$ and $p<0.05$ were identified as significant difference.

\subsection{Statistical analysis}


Data are presented as the mean \pm SD. A significance level of $p<0.05$ was used for all tests. Differences between two means were assessed by unpaired and two-tailed Student's t-test. p values $<0.05$ were considered statistically significant.

\section{Results}

\subsection{Long-term alcohol administration induces variation of lipidome in PFC and striatum}

The lipid profiling of PFC and striatum, the two brain regions which are closely associated with alcohol addiction, was measured after the rats were administrated with alcohol for one year. The lipid mixtures were separated by liquid chromatography and the representative total ion current (TIC) chromatograms of both PFC and striatum were shown in Fig. 1. The marked peaks were major lipids categories identified through mass spectrometry, and the different lipids classes were observed in positive and negative ionization modes. Thus, differential lipids were analyzed and identified based on both ion modes. We observed that long-term alcohol exposure caused obvious differences in some peaks of TIC chromatograms, suggesting alcohol may significantly modify lipidomic profiles of brain.

Fig. 1. The presentative TIC chromatograms obtained from PFC (A) and striatum (B) region of rats administrated with or without alcohol. The positive ion (ESI+) and negative ion (ESI-) were displayed in red and blue color, respectively.

\subsection{OPLS-DA model analysis of brain lipidomic changes caused by alcohol}

To identify lipidomics differences between water- and alcohol-treated rats, the supervised orthogonal partial least squares discriminant analysis (OPLS-DA) model was constructed to visualize the distribution of two groups. The score plots from OPLS-DA model of both brain regions displayed a clear differentiation between water- and alcohol-treated groups (Fig. 2A and $2 \mathrm{~B}$ ). The quality and effectiveness of the constructed model were evaluated using the goodness of fitness $\left(\mathrm{R}^{2}\right)$ and the predictability $\left(\mathrm{Q}^{2}\right)$. The $\mathrm{R}^{2}$ value of all models is more than 
0.5 , indicating good fitness. Thus, the variable importance (VIP) value of $\geq 1$ was identified as the significant changed $\mathrm{m} / \mathrm{z}$ mediating the clustering of groups. In PFC, 430 and $302 \mathrm{of} \mathrm{m} / \mathrm{z}$ ratios were showed $\mathrm{VIP} \geq 1$ in positive and negative ion mode, respectively (Fig. 2C). Among all detected $\mathrm{m} / \mathrm{z}$ ratios in both modes, 380 of ratios in total were significant changed $(p<0.05)$ in alcohol group in comparison to water group (Fig. 2D). In striatum tissue, $743 \mathrm{of} \mathrm{m} / \mathrm{z}$ ratios, 487 in positive ion detection mode and 256 in negative ion detection mode, were displayed VIP $\geq 1$ (Fig. 2E). The statistical analysis showed that alcohol administration caused significant changes in 243 of $\mathrm{m} / \mathrm{z}$ ratios in stratum (Fig.2F). Collectively, these results suggested that some lipid metabolites in PFC and striatum regions were profoundly modified by long-term alcohol exposure.

Fig. 2. Pattern recognition analysis reveals that chronic alcohol exposure causes significant changes in lipidome profiling of brain. (A and B) 3D OPLS-DA scores scatter plot displays significant difference of PFC (A) and striatum (B) between control and alcoholtreated rats. The model parameters $\mathrm{R}^{2}$ shows variance and $\mathrm{Q}^{2}$ indicates the excellent quality of model. (C and E) S-plot illustrates the putative $\mathrm{m} / \mathrm{z}$ ratio (VIP $\geq 1$, red color) responsible for the discrimination of control and alcohol-treated rats. (D and F) Volcano plot of mass features was constructed with $\mathrm{x}$-axis presenting fold change (logarithms of alcohol-to-control ratios) and y-axis significance level (logarithms of $p$ value). The significantly changed lipid species $(p<0.05)$ were marked with color, and those metabolites without obvious change were shown in black dots. Red and blue color show the significantly increased and decreased lipids species in alcohol-treated rats, separately. In PFC region, $n=7$ samples for control group and $\mathrm{n}=10$ samples for alcohol-treated male rats. In striatum region, $\mathrm{n}=10$ samples for control group and $n=10$ samples for alcohol-treated male rats.

\subsection{Chronic alcohol consumption causes marked alteration in lipid profiles of brain}

To determine alcohol-modified lipids, those $\mathrm{m} / \mathrm{z}$ ratios with $\mathrm{VIP} \geq 1$ and $p<0.05$ were 
considered as significantly changed lipids induced by chronic alcohol exposure. The results showed that 110 lipids in PFC (Supplemental table 1) and 79 lipids in striatum were significantly altered in alcohol-treated rats in comparison to water-treated rats (Supplemental table 2). Among these distinguished lipids, 36 lipids were detected in both brain regions. Heat map displayed change degree of those altered lipids (Fig. 3A, Supplemental table 1 and table 2). Overall, alcohol caused up-regulation of the majority of lipids in PFC and striatum. Except for LacCer (d34:0) that presented conversely change, all other distinguished lipids presented the similar modified trend with most of profoundly enhanced in response to alcohol. Notably, the significantly altered lipids included diacylglycerol (DG), phosphatidylserine (PS), phosphatidylcholine (PC), phosphatidylethanolamine (PE) and fatty acyls (FA) (Fig. 3B). Considering lipid class, all significantly modified lipids were classified into 5 groups, glycerophospholipid (GP), glycerolipid (GL), fatty acyls (FA), sphingolipids (SP) and sterol lipids (ST). The most modified lipids were PS, PC and PE, which are classified into GP, accounting for the most proportion of those modified lipids induced by alcohol. In addition, the other two major classes GL and FA exhibited 34\% of total lipid groups in both brain regions (Fig.3C). Taken together, the similar modification of the lipids in PFC and striatum suggested that long-term alcohol consumption can result in universal disturbance to brain lipid metabolism.

Fig. 3. Chronic alcohol modifies lipidome in the PFC and striatum. (A) The heat maps show all profoundly modified lipid species (VIP $\geq 1, p<0.05$ ). The color bars represent the value of the $\log 2$-fold changed ratio of each lipid species. Statistical analysis for individual lipid species was performed based on the unpaired method. (B) The 10x10 dot plot shows the ratio of each lipids species among the total identified differential lipids species. (C) Pie chart displays the distribution of identified lipid species when classified into corresponding lipid class. 


\subsection{Endoplasmic reticulum may be the main organelle of chronic alcohol exposure}

ER is the main organelle that produces the bulk of structural phospholipids and cholesterol for cells. The major species of GP class, PC, PE, phosphatidylinositol (PI), PS and phosphatidic acid (PA), are mainly synthesized in ER (Röhrl and Stangl 2018; Volkmar et al. 2019; Yang, Lee, and Fairn 2018). Some species of SP and ST groups are also biosynthesized in ER. Except for ER, Golgi, plasma membrane and mitochondria are also responsible for the synthesis of some widely-distributed or organelle-specific lipids (Preissl et al. 2018). To determine what kind of organelle was the mostly affected by long-term alcohol exposure, the synthesized lipidomic profiling of PFC and striatum were analyzed in plasma membrane and different organelles, including ER, mitochondria and Golgi. All putative synthesized lipids were displayed in Fig. 4. Alcohol exposure activated the catalytic metabolism of lipids in analyzed organelles as well as in plasma membrane. Notably, most of profoundly distinguished lipids were traced back to ER-dependent synthetic pathway (Fig. 4A). However, less numbers lipids were influenced by alcohol in mitochondria, Golgi and plasma membrane (Fig. 4B-4D). Overall, these results indicate that long-term alcohol administration markedly affected lipid synthesis, especially those lipids produced by ER.

Fig. 4. The classification of main site responsible for synthesizing identified differential lipids. The major sites for lipid synthesis include endoplasmic reticulum (A), mitochondria (B), Golgi (C) and plasma membrane (D). In alcohol-treated groups, the relatively fold change of corresponding lipids species in both PFC (red) and striatum (blue) region was referred to the control group. Error bars represent the mean \pm SD. $* p \leq 0.05, * * p \leq 0.01$ and $* * * p \leq 0.001$, Student's t-test.

\subsection{Chronic alcohol exposure significantly modifies lipid fatty acyl chain profiling}

Homeostasis of cellular membrane is pivotal for the maintenance of normal cell functions in the brain. Abnormal length of lipid fatty acyl chain, such as changed carbon length or 
saturation degree, can impair neuronal functioning by modulating the lipid raft in membrane (Lesa 2003; Raghupathy et al. 2015). To systematically evaluate the influences of alcohol on lipidomic profiling, the pattern of fatty-acyl chain of diacyl-GPs and DG species were further analyzed. As shown in Fig. 5A, the fatty acyl chain was evidently remodeled by alcohol exposure, and the majority of modified GPs and DG in both PFC and striatum regions composed of 36 carbons or more total carbons. Notably, the increased length of fatty acyl chain contained more unsaturated bonds in alcohol-exposed rats (Fig. 5B). As the long-chain polyunsaturated phospholipids prefer to form highly organized lamellar microdomain membrane structures and determine the sensory neuronal cells (Paolo and Kim 2012; Wassall and Stillwell 2009), the increase of long-chain polyunsaturated phospholipids may participate neuropathological process caused by chronic alcohol exposure.

Fig. 5. The alternation of lipid fatty acid composition induced by alcohol. (A) Relative variation of fatty acid carbon chain length both in PFC (red) and striatum (blue) region. (B) The total degree of unsaturation both in the PFC (Red) and striatum (blue) region. Error bars represent the mean \pm SD. ${ }^{*} p \leq 0.05,{ }^{* *} p \leq 0.01$ and $* * * p \leq 0.001$, Student's t-test.

\section{Discussion}

Alcohol drinking causes serious health problems and long-time abuse even leads to irreversible alteration in brain structure and function (Kranzler and Soyka 2018; Rehm et al. 2009). Particularly, PFC and striatum, two critical regions involving in cognitive function, are vulnerable to neurotoxic damage. (Johnson, O’Callaghan, and Miller 2002; Luikinga, Kim, and Perry 2018; Schmitz et al. 2017). So far, brain damage or degeneration has been widely found in the vast majority of chronic alcoholics; however, the underlying molecular neuropathology has not yet been fully determined. In this study, the effect of long-term 
alcohol exposure on PFC and striatum were comprehensively analyzed by LC-MS/MS-based lipidomics approach. The results show that alcohol widely and profoundly alters the lipidomics of brain, displaying abnormal lipid accumulation in both detected brain regions.

Among the widely modified lipidome, GP, GL and FA were observed the most preferentially modified lipids classes. Interestingly, the majority of those differentially modified lipids are involved in ER-related catalytic pathways, suggesting that chronic alcohol exposure imposes ER stress for the brain. Except for the abnormal accumulation of lipid species in cells, the enhanced length and unsaturation degree of carbon chain further augmented ER stress. Chronic ER stress indeed has ability to disturb intensity of membrane structures and also fundamental organelle functions, such as mitochondria, Golgi, nucleus (Fig. 6). To the best of our knowledge, this is the first report showing that chronic alcohol exposure significantly alters the lipidomic profile of PFC and striatum, which may underlie the potential pathogenesis of alcohol-related neurotoxicity and neuroplasticity.

Fig. 6. A putative schematic model of chronic alcohol exposure-induced the disruption of cellular metabolism.

Alcoholics with cognitive impairment often accompany with deficiency in both white matter and gray matter brain structure (Johnson et al. 2002). The correlation between the degree of brain atrophy and the rate of amount of consumed alcohol have been widely known; however, the exact neuropathological mechanism still remains unclear. Using the lipidomic analytical approaches, we revealed that some species belonging to GPs were significantly altered in both PFC and striatum of alcohol-exposed rats. GPs are the key components of membrane structure and their composition vary a lot among different cell types, organelles, and inner/outer membranes in mammalian mitochondria due to their divergent biological functions (De et al. 2018; Klaming et al. 2019). As GPs provide neural membranes with stability, fluidity and permeability, they are required for normal biological function of integral 
membrane proteins, receptors, and ion-channels. Thinking above, our results suggest that chronic alcohol exposure may affect brain function possibly through altering GPs composition. In fact, alterations of GPs composition in neural membrane have been reported to involve in neurological disorders (Armbruster et al. 2008; Daisuke Hishikawa et al. 2014; Jiang et al. 2017). In addition, because myelin contains the highest content of GPs among the membranes of brain, we thus speculate that the majority changed GPs may lead to disruption of myelin fiber and even neuron injury.

Most GPs are synthesized via two synthetic pathways called the Kennedy pathway and the cytidine diphosphate-diacylglycerol (CDP-DAG) pathways (Daisuke Hishikawa et al. 2014). In the CDP-DAG pathway, phospholipid synthesis strongly depends on phospholipid transport between ER and mitochondria as well as between the mitochondrial outer and inner membranes (Henry, Kohlwein, and Carman 2012; Tamura, Sesaki, and Endo 2014). Phosphatidic acid is the starting material for the synthesis of all other phospholipids and is mainly produced in two ways. Firstly, using glycerol-3-phosphate or dihydroxyacetone phosphate as material to generate PA catalyzing by an ER-resident enzyme (Osman, Voelker, and Langer 2011; Scharwey n.d.). Secondly, DG is phosphorylated to form PA by the DG kinase Dgk1 in the ER (Tamura et al. 2014). In this study, we found that long-term alcohol exposure significantly altered PA and PA-related phospholipids, suggesting that alcohol might activate lipogenesis-related enzymes and thus simulate the synthetic pathways of lipids, eventually causing the aberrant accumulation of lipids species.

The maintenance of all aspects of fatty acids and lipid homeostasis are executed by ER, which is the hub organelle of protein and lipid synthesis, membrane biogenesis, xenobiotic detoxification as well as calcium homeostasis (Athenstaedt and Biomembrane 1997). Thus, the perturbation of ER homeostasis poses major challenges to lipid metabolism that ultimately can lead to ER stress and metabolic dysfunctions (Fu et al. 2011). In addition, the 
transformation of saturated fatty acids to unsaturated fatty acids can promote ER stress and lipotoxicity in cells and animals (Fu, Watkins, and Hotamisligil 2012). ER stress activates inflammatory cascades, such as JNK/IKK/CREB pathways, boosts ROS generation and leads the overload of mitochondrial calcium storage (Flowers et al. 2008; Green and Olson 2011). The excessive oxidative stress in mitochondria triggers the opening of mitochondrial permeability transition pore and finally induce the collapse of mitochondrial membrane potential, activating the cell death related pathways (Fu et al. 2012). In this study, alcohol exposure caused accumulation of long length of unsaturated lipids species, which might facilitate mitochondrial ROS production and further cell death. In fact, alcohol-induced ER stress and disruption of cellular protein homeostasis have been established as an important mechanism contributing to liver diseases.

More interestingly, the enhanced lipids species especially GP displayed longer fatty acyl chain and higher unsaturation degree in the present study. Indeed, the increase number of double bonds is positively associated with the susceptibility of fatty acids to free radical damage. For example, the short length of carbon chain with one double bond $18: 1 n-9$ is relatively more stable to the impairment of oxidative stress than the long length of carbon chain with 6 double bonds 22:6n-3. Various studies have shown that increased degree of fatty acid unsaturation boosts oxidative damage to lipids and proteins as well as the generation rates of mitochondrial superoxide (Angelova and Abramov 2018; Xia et al. 2018). Conversely, the lower double bond content leads to a lower sensitivity to oxidative induced lipid peroxidation. The process of lipid peroxidation damages not only the lipids but also tissue proteins and DNA through the final products-generated secondary radicals, such as malondialdehyde. This secondary damage has been widely demonstrated crucial for disease, such as aging (Pamplona et al. 2004). Therefore, we speculated that the high degree of unsaturated fatty acids may cause the brain DNA, proteins, lipids and even cell organelle, 
such as mitochondria, more susceptible to alcohol-induced oxidative damage.

In this study, alcohol-modified pattern of lipidomics in brain is consistent with recent reports. For instance, alcohol exposure upregulates the expression of acid sphingomyelinase (ASMase), which is capable to induce ER-stress and consequently activates the lipogenic pathways through synthesizing ceramide (Pamplona et al. 2004). Ceramides make important contributions to cell membrane structure and exert diverse fundamental cellular process, including cell growth, proliferation, motility, adhesion, differentiation, senescence, and apoptosis (Fernandez et al. 2013). Increased ceramide can induce lipotoxicity and ER stress, which involves in the pathogenesis of several neurodegenerative diseases (Contreras et al. 2014). Interestingly, among those modified lipids of alcohol-exposed brain, we noticed that ceramide level was up-regulated in PFC, suggesting that ER in brain might undergo the stress during alcohol exposure. Considering the significantly altered lipids, which are synthesized in ER, as well as the lipid patterns (Fig. 4), we speculated that long-term alcohol exposure may modify brain lipidomics through ER stress. Moreover, the altered lipidome profiling can interrupt the stability of cell membrane rafts or change the neuroplasticity, which may further damage the integrity of brain tissue and consequently cause cognitive impairment. Further research is needed to investigate which metabolic enzymes or the well-known ASMase mediate alcohol-induced dysfunction of lipidome profiling in brain.

Collectively, by using lipidomics approach, we find that chronic alcohol exposure induces a profound alteration in the lipidome of PFC and striatum. Although the lipid landscape of the brain is complex and highly dynamic, specific lipid classes appear to be involved in alcoholinduced neuropathology. The predominantly modified lipid species include PS, PC and PE, which are classified into GP. The availability of lipid profiles provides a new insight into the neurotoxicity caused by chronic alcohol exposure. 1 


\section{Acknowledgments}

This work was partially supported by National Natural Science Foundation of China (Grants 81571301, 81871043, 81271467, 81272459), the National Science and Technology Major Project (2018ZX09201017), and “1·3·5 Project for Disciplines of Excellence, West China Hospital, Sichuan University”. 


\section{References}

Allen, John A., Robyn A. Halverson-tamboli, and Mark M. Rasenick. 2007. "Lipid Raft Microdomains and Neurotransmitter Signalling." 8(FEBRUARY):128-40.

Angelova, Plamena R. and Andrey Y. Abramov. 2018. "Role of Mitochondrial ROS in the Brain: From Physiology to Neurodegeneration." FEBS Letters 592(5):692-702.

Armbruster, Blaine N. et al. 2008. "Membrane Lipids: Where They Are and How They Behave." Nat Rev Mol Cell Biol 9(2):112-14.

Athenstaedt, Karin and S. F. B. Biomembrane. 1997. "Biosynthesis of Phosphatidic Acid in Lipid Particles and Endoplasmic Reticulum of Saccharomyces Cerevisiae." 179(24):7611-16.

Becker, Howard C. and Dorit Ron Guest. 2014. "Animal Models of Excessive Alcohol Consumption : Recent Advances and Future Challenges." 48:205-8.

Boggs, J. M. 2006. "Myelin Basic Protein : A Multifunctional Protein.” 63:1945-61.

Castro-perez, Jose M. et al. 2010. "Comprehensive LC - MS E Lipidomic Analysis Using a Shotgun Approach and Its Application to Biomarker Detection and Identification in Osteoarthritis Patients Research Articles." 2377-89.

Cederbaum, Arthur I. and Æ. Yongke Lu. 2009. "Role of Oxidative Stress in AlcoholInduced Liver Injury." 519-48.

Chan, Robin B. et al. 2012. "Comparative Lipidomic Analysis of Mouse and Human Brain with Alzheimer Disease * $\square . "$ 287(4):2678-88.

Cui, Jiahui et al. 2019. "Acetaldehyde Induces Neurotoxicity In Vitro via Oxidative Stressand Ca 2 + Imbalance-Mediated Endoplasmic Reticulum Stress.” 2019.

Daisuke Hishikawa, Tomomi Hashidate, Takao Shimizu, and Hideo Shindou. 2014. Diversity and Function of Membrane Glycerophospholipids Generated by the Remodeling Pathway in Mammalian Cells.

De, Suzanne M. et al. 2018. "Imaging Mass Spectrometry of Frontal White Matter Lipid Changes in Human Alcoholics." Alcohol 67:51-63.

Deak, Joseph D., Alex P. Miller, and Ian R. Gizer. 2019. "Genetics of Alcohol Use Disorder: A Review." Current Opinion in Psychology 27:56-61.

Dey, Aparajita and Arthur I. Cederbaum. 2006. "Alcohol and Oxidative Liver Injury." 63-74.

Domnina, N. S., A. A. Fenin, V. I. Ermakov, and E. P. Magomedbekov. 2015. "Interaction of Anthocyanins and Anthocyanidins with $\alpha$ Hydroxyethyl Radicals." 49(6):388-93.

Erickson, Emma K., Emily K. Grantham, Anna S. Warden, and Adron Harris. 2018. "Neuroimmune Signaling in Alcohol Use Disorder." Pharmacology, Biochemistry and Behavior \#pagerange\#.

Ermilova, Inna and Alexander P. Lyubartsev. 2019. "Cholesterol in Phospholipid Bilayers: Positions and Orientations inside Membranes with Different Unsaturation Degrees." Soft Matter 15(1):78-93.

Fabelo, Noemí et al. 2011. "Severe Alterations in Lipid Composition of Frontal Cortex Lipid Rafts from Parkinson' s Disease and Incidental Parkinson' s." Molecular Medicine $7(12)$.

Fernandez, Anna et al. 2013. "ASMase Is Required for Chronic Alcohol Induced Hepatic Endoplasmic Reticulum Stress and Mitochondrial Cholesterol Loading." Journal of Hepatology 59(4):805-13.

Ferrer, Isidre. 2009. "Progress in Neurobiology Early Involvement of the Cerebral Cortex in Parkinson' s Disease : Convergence of Multiple Metabolic Defects." 88:89-103.

Flowers, Matthew T. et al. 2008. "Liver Gene Expression Analysis Reveals Endoplasmic Reticulum Stress and Metabolic Dysfunction in SCD1-Deficient Mice Fed a Very LowFat Diet." Physiological Genomics.

$\mathrm{Fu}$, Suneng et al. 2011. "Aberrant Lipid Metabolism Disrupts Calcium Homeostasis Causing 
Liver Endoplasmic Reticulum Stress in Obesity." Nature.

Fu, Suneng, Steven M. Watkins, and Gökhan S. Hotamisligil. 2012. "The Role of Endoplasmic Reticulum in Hepatic Lipid Homeostasis and Stress Signaling." Cell Metabolism 15(5):623-34.

Gao, Hong Lei et al. 2018. "High-Throughput Lipidomics Characterize Key Lipid Molecules as Potential Therapeutic Targets of Kaixinsan Protects against Alzheimer's Disease in APP/PS1 Transgenic Mice." Journal of Chromatography B: Analytical Technologies in the Biomedical and Life Sciences 1092(June):286-95.

Green, Christopher D. and L. Karl Olson. 2011. "Modulation of Palmitate-Induced Endoplasmic Reticulum Stress and Apoptosis in Pancreatic $\beta$-Cells by Stearoyl-CoA Desaturase and Elov16." American Journal of Physiology-Endocrinology and Metabolism.

Harper, Clive. 1998. "The Neuropathology of Alcohol-Specific Brain Damage, or Does Alcohol Damage the Brain?" Journal of Neuropathology and Experimental Neurology 57(2):101-10.

Harper, Clive. 2009. “The Neuropathology of Alcohol-Related Brain Damage.” 44(2):136-40. He, Xiaohua, Edith V Sullivan, Roger K. Stankovic, Clive G. Harper, and Adolf Pfefferbaum. 2007. "Interaction of Thiamine Deficiency and Voluntary Alcohol Consumption Disrupts Rat Corpus Callosum Ultrastructure." 1:2207-16.

Henry, Susan A., Sepp D. Kohlwein, and George M. Carman. 2012. "Metabolism and Regulation of Glycerolipids in the Yeast Saccharomyces Cerevisiae." 190(February):317-49.

Holthuis, Joost C. M. and Anant K. Menon. 2014. "Lipid Landscapes and Pipelines in Membrane Homeostasis."

Inoue, Yuki, Hirokazu Hara, Yukari Mitsugi, Eiji Yamaguchi, and Tetsuro Kamiya. 2018. "Hydroxydopamine-Induced Cell Death via Activation of Nrf2-ARE And." Neurochemistry International 112:288-96.

Jiang, Linhong et al. 2017. "Remodeling of Brain Lipidome in Methamphetamine-Sensitized Mice.” Toxicology Letters 279(April):67-76.

Johnson, Elizabeth Anne, James P. O'Callaghan, and Diane B. Miller. 2002. "Chronic Treatment with Supraphysiological Levels of Corticosterone Enhances D-MDMAInduced Dopaminergic Neurotoxicity in the C57BL/6J Female Mouse." Brain Research 933(2):130-38.

Klaming, Ruth et al. 2019. "Shared Gray Matter Reductions across Alcohol Use Disorder and Posttraumatic Stress Disorder in the Anterior Cingulate Cortex : A Dual Meta-Analysis." 10(August 2018).

Kranzler, Henry R. and Michael Soyka. 2018. "Diagnosis and Pharmacotherapy of Alcohol Use Disorder a Review." JAMA - Journal of the American Medical Association 320(8):815-24.

Kulkarni, Hemant, Manju Mamtani, John Blangero, and Joanne E. Curran. 2017. "Lipidomics in the Study of Hypertension in Metabolic Syndrome." Current Hypertension Reports 19(1):1-8.

De La Monte, Suzanne M. and Jillian J. Kril. 2014. "Human Alcohol-Related Neuropathology." Acta Neuropathologica 127(1):71-90.

Lesa, G. M. 2003. "Long Chain Polyunsaturated Fatty Acids Are Required for Efficient Neurotransmission in C. Elegans." Journal of Cell Science 116(24):4965-75.

Li, Hongchun et al. 2018. "Lipidomic Signature of Serum from the Rats Exposed to Alcohol for One Year." Toxicology Letters 294(March):166-76.

Links, Dynamic Article. 2011. "Molecular BioSystems Application of Plasma Lipidomics in Studying the Response of Patients with Essential Hypertension to Antihypertensive Drug 
Therapy W.” 3271-79.

Luikinga, Sophia J., Jee Hyun Kim, and Christina J. Perry. 2018. "Developmental Perspectives on Methamphetamine Abuse: Exploring Adolescent Vulnerabilities on Brain and Behavior." Progress in Neuro-Psychopharmacology and Biological Psychiatry 87(May 2017):78-84.

Marin, Raquel et al. 2017. "Neurobiology of Aging Anomalies Occurring in Lipid pro Fi Les and Protein Distribution in Frontal Cortex Lipid Rafts in Dementia with Lewy Bodies Disclose Neurochemical Traits Partially Shared by Alzheimer' $\mathrm{s}$ and Parkinson' $\mathrm{s}$ Diseases." 49:52-59.

Matyash, Vitali, Gerhard Liebisch, Teymuras V. Kurzchalia, Andrej Shevchenko, and Dominik Schwudke. 2008. "Lipid Extraction by Methyl- Tert -Butyl Ether for HighThroughput Lipidomics." Journal of Lipid Research 49(5):1137-46.

Mayfield, J., M. A. Arends, R. A. Harris, and Y. A. Blednov. 2016. Genes and Alcohol Consumption: Studies with Mutant Mice. 1st ed. Elsevier Inc.

Nakamura, Kazuhiko et al. 2003. "Acetaldehyde Adducts in the Brain of Alcoholics." 591-93. Nutabi, Anne-lieke F. Van Deijk et al. 2017. "Astrocyte Lipid Metabolism Is Critical for Synapse Development and Function in Vivo." (September 2016):670-82.

Osman, Christof, Dennis R. Voelker, and Thomas Langer. 2011. "Making Heads or Tails of Phospholipids in Mitochondria." 192(1).

Pamplona, Reinald and Gustavo Barja. 2001. "Effect of the Degree of Fatty Acid Unsaturation of Rat Heart Mitochondria on Their Rates of $\mathrm{H} 2 \mathrm{O} 2$ Production and Lipid and Protein Oxidative Damage." 122:427-43.

Pamplona, Reinald, Manel Portero-otın, Alberto Sanz, J. Requena, and Gustavo Barja. 2004. "Modification of the Longevity-Related Degree of Fatty Acid Unsaturation Modulates Oxidative Damage to Proteins and Mitochondrial DNA in Liver and Brain." 39:725-33.

Paolo, Gilbert Di and Tae-wan Kim. 2012. "347. Linking Lipids to Alzheimer' s Disease : Cholesterol and Beyond." Aging 12(5):284-96.

Parry, Charles D., Jayadeep Patra, and Jürgen Rehm. 2011. "Alcohol Consumption and NonCommunicable Diseases :" 1718-24.

Pfefferbaum, Adolf et al. 2006. "Longitudinal Brain Magnetic Resonance Imaging Study of Chronic Alcohol Consumption." 30(7):1248-61.

Preissl, Sebastian et al. 2018. "Single-Nucleus Analysis of Accessible Chromatin in Developing Mouse Forebrain Reveals Cell-Type-Specific Transcriptional Regulation." Nature Neuroscience 21(3):432-39.

Raghupathy, Riya et al. 2015. "Transbilayer Lipid Interactions Mediate Nanoclustering of Lipid-Anchored Proteins." Cell 161(3):581-94.

Ramírez, Cristina M. et al. 2009. "Molecular and Cellular Neuroscience VDAC and ER $\alpha$ Interaction in Caveolae from Human Cortex Is Altered in Alzheimer ' s Disease." Molecular and Cellular Neuroscience 42(3):172-83.

Rehm, Jürgen et al. 2010. "The Relation between Different Dimensions of Alcohol Consumption and Burden of Disease : An Overview." (April 2009):817-43.

Rehm, Jürgen and D. Ph. 2012. "Chronic Diseases and Alcohol Use.” 155-73.

Röhrl, Clemens and Herbert Stangl. 2018. "Cholesterol Metabolism - Physiological Regulation and Pathophysiological Deregulation by the Endoplasmic Reticulum." 28085.

Scharwey, Melanie. n.d. "Mitochondrial Lipid Transport at a Glance."

Schmitz, Felipe. et al. 2017. "Methylphenidate Decreases ATP Levels and Impairs Glutamate Uptake and $\mathrm{Na}+\mathrm{K}+-\mathrm{ATPase}$ Activity in Juvenile Rat Hippocampus." Molecular Neurobiology 54(10):7796-7807.

Schuchman, Edward H. 2010. "Acid Sphingomyelinase, Cell Membranes and Human Disease: 
Lessons from Niemann-Pick Disease.” FEBS Letters 584(9):1895-1900.

Schweikl, H. et al. 2016. "Critical Role of Superoxide Anions and Hydroxyl Radicals in HEMA-Induced Apoptosis." Dental Materials 33(1):110-18.

Shevchenko, Andrej and Kai Simons. 2010. "Lipidomics: Coming to Grips with Lipid Diversity." Nature Publishing Group 11(8):593-98.

SMITH, ROSS. 1977. "NON-COVALENT CROSS-LINKING OF LIPID BILAYERS BY MYELIN BASIC PROTEIN A." 470:170-84.

Tamura, Yasushi, Hiromi Sesaki, and Toshiya Endo. 2014. "Phospholipid Transport via Mitochondria." 1(10).

Volkmar, Norbert et al. 2019. "The ER Membrane Protein Complex Promotes Biogenesis of Sterol-Related Enzymes Maintaining Cholesterol Homeostasis."

Want, Elizabeth J. et al. 2013. "Global Metabolic Profiling of Animal and Human Tissues via UPLC-MS." Nature Protocols 8(1):17-32.

Wassall, Stephen R. and William Stillwell. 2009. "Polyunsaturated Fatty Acid-Cholesterol Interactions: Domain Formation in Membranes." Biochimica et Biophysica Acta Biomembranes 1788(1):24-32.

Wenk, Markus R. 2005. "THE EMERGING FIELD OF LIPIDOMICS.” 4(July):594-610.

Worley, B. and R. Powers. 2013. "Multivariate Analysis in Metabolomics." Current Metabolomics 1(1):92-107.

Worley, Bradley and Robert Powers. 2016. "PCA as a Practical Indicator of OPLS-DA Model Reliability." Current Metabolomics 4(2):97-103.

Xia, Wenxiang, Jincheng Li, Xilai Zheng, and Guizhi Wu. 2018. "Isolation and Biodegradation Characteristic of Petroleum Degrading Bacteria from Jiaozhou Bay." 3rd International Conference on Bioinformatics and Biomedical Engineering, ICBBE 2009 592:743-58.

Yan, Furong, Hong Zhao, and Yiming Zeng. 2018. "Lipidomics: A Promising Cancer Biomarker." Clinical and Translational Medicine 7(1):21.

Yang, Yanbo, Minhyoung Lee, and Gregory D. Fairn. 2018. "Cro Phospholipid Subcellular Localization and Dynamics." 293:6230-40.

Zahr, Natalie M., Kimberley L. Kaufman, and Clive G. Harper. 2011. "Clinical and Pathological Features of Alcohol-Related Brain Damage." Nature Publishing Group 7(5):284-94.

Zheng, Mengge et al. 2018. "Effects of Chain Length and Degree of Unsaturation of Fatty Acids on Structure and in Vitro Digestibility of Starch-Protein-Fatty Acid Complexes." Journal of Agricultural and Food Chemistry 66(8):1872-80.

Zhou, Xinchun et al. 2012. "Identification of Plasma Lipid Biomarkers for Prostate Cancer by Lipidomics and Bioinformatics." 7(11). 
A
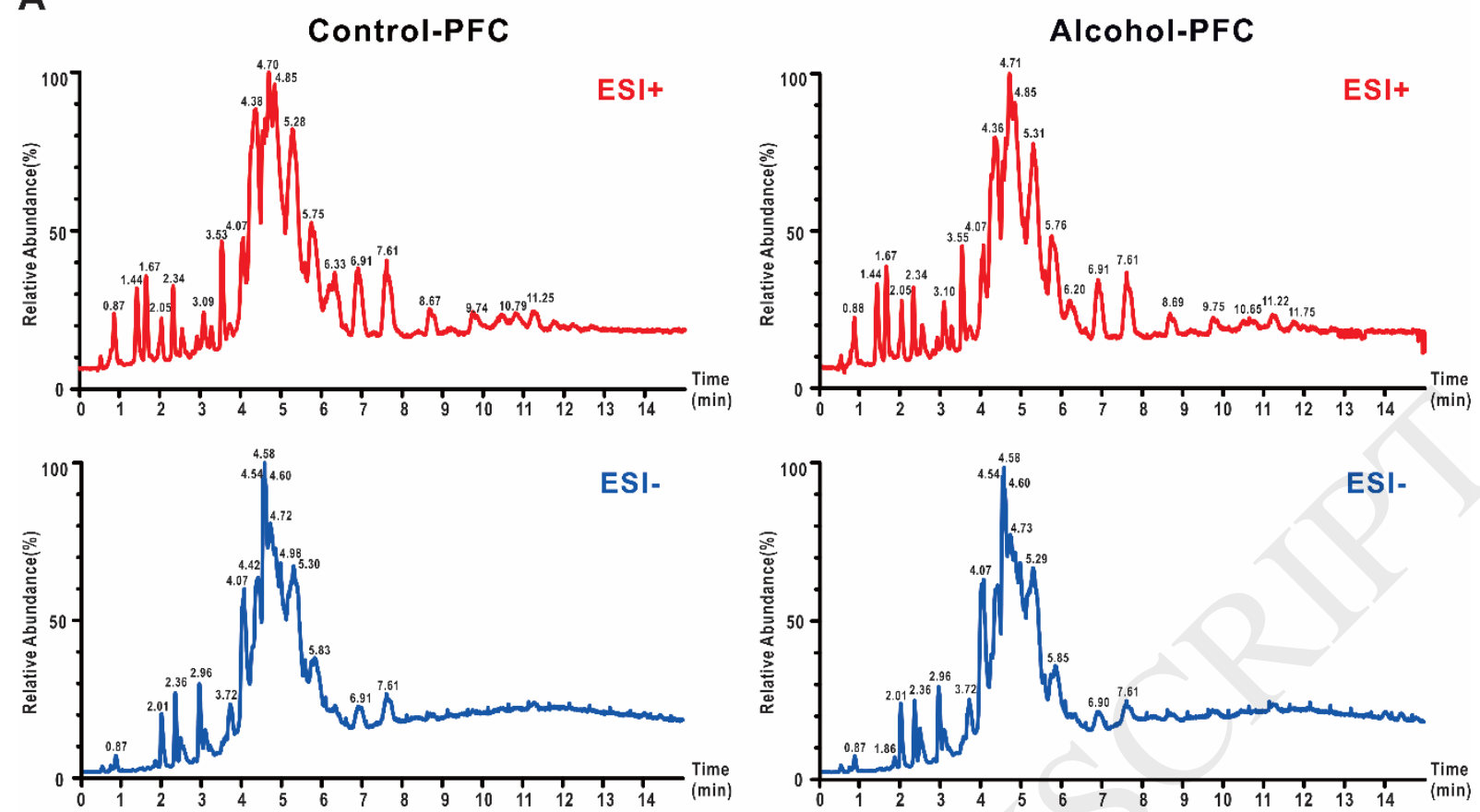

B
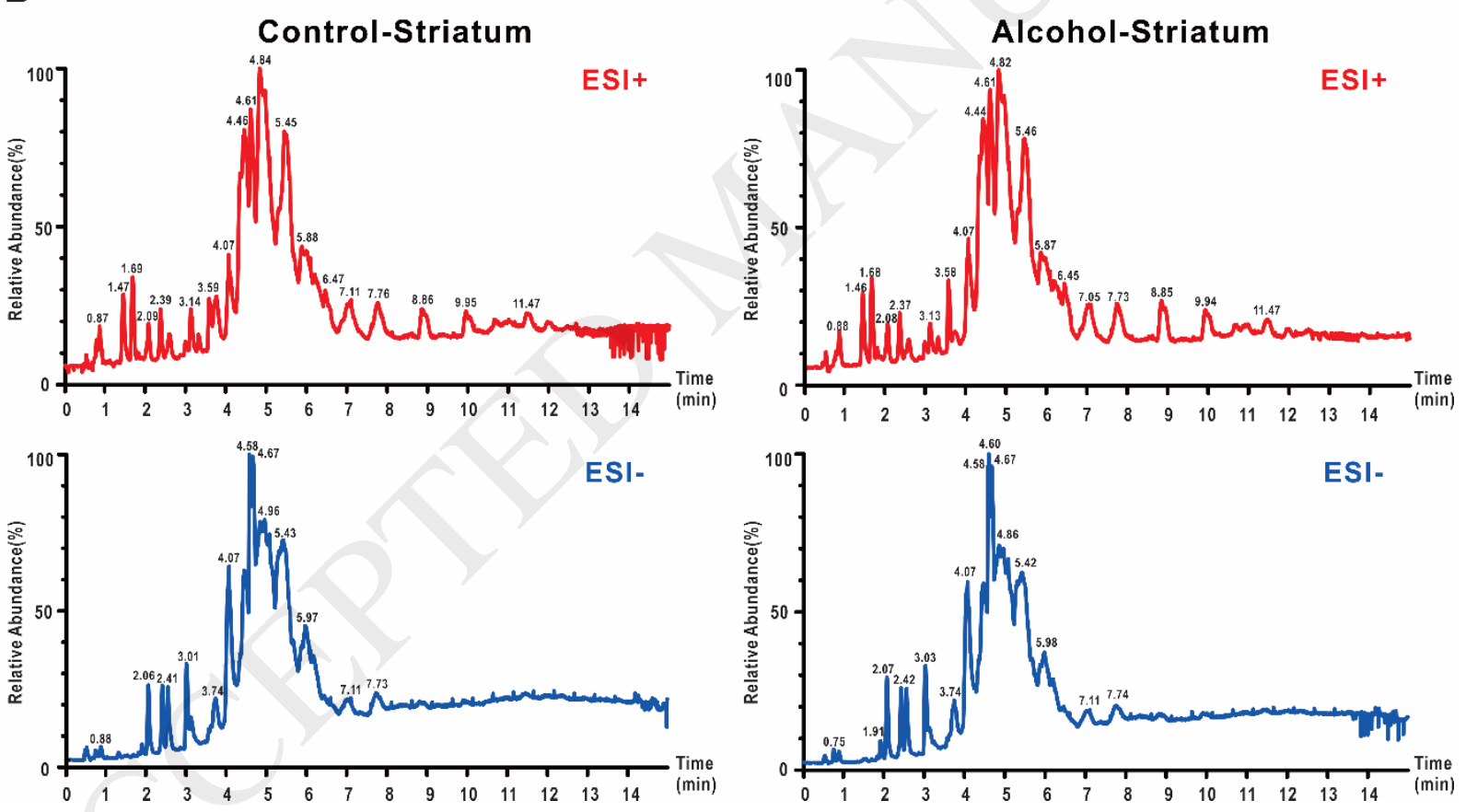
A
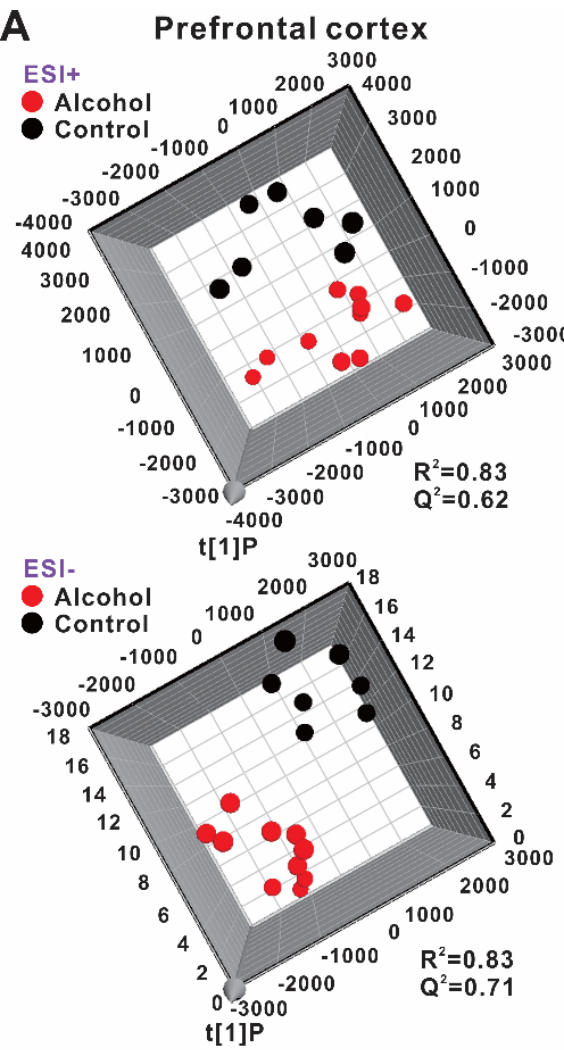

B
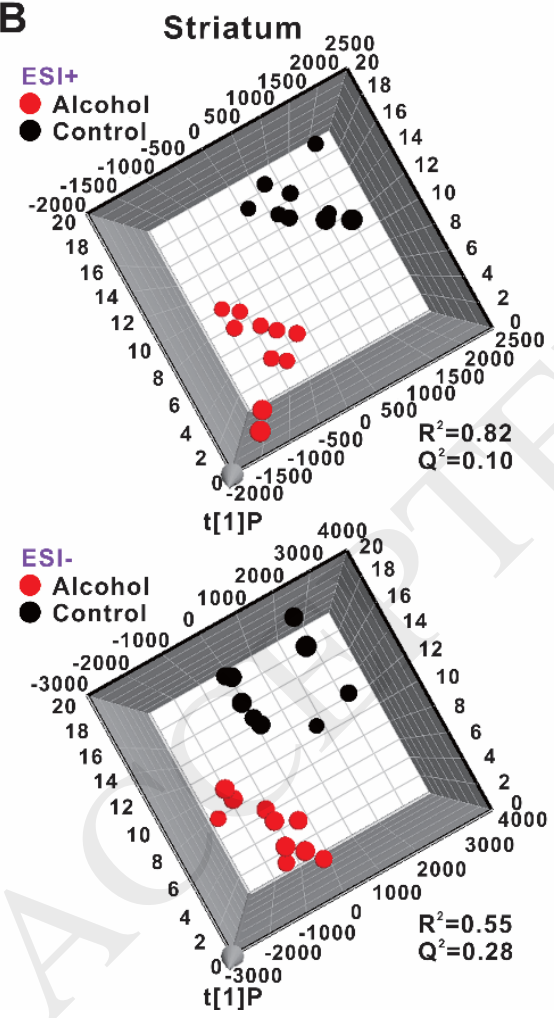

C Prefrontal cortex

ESI+
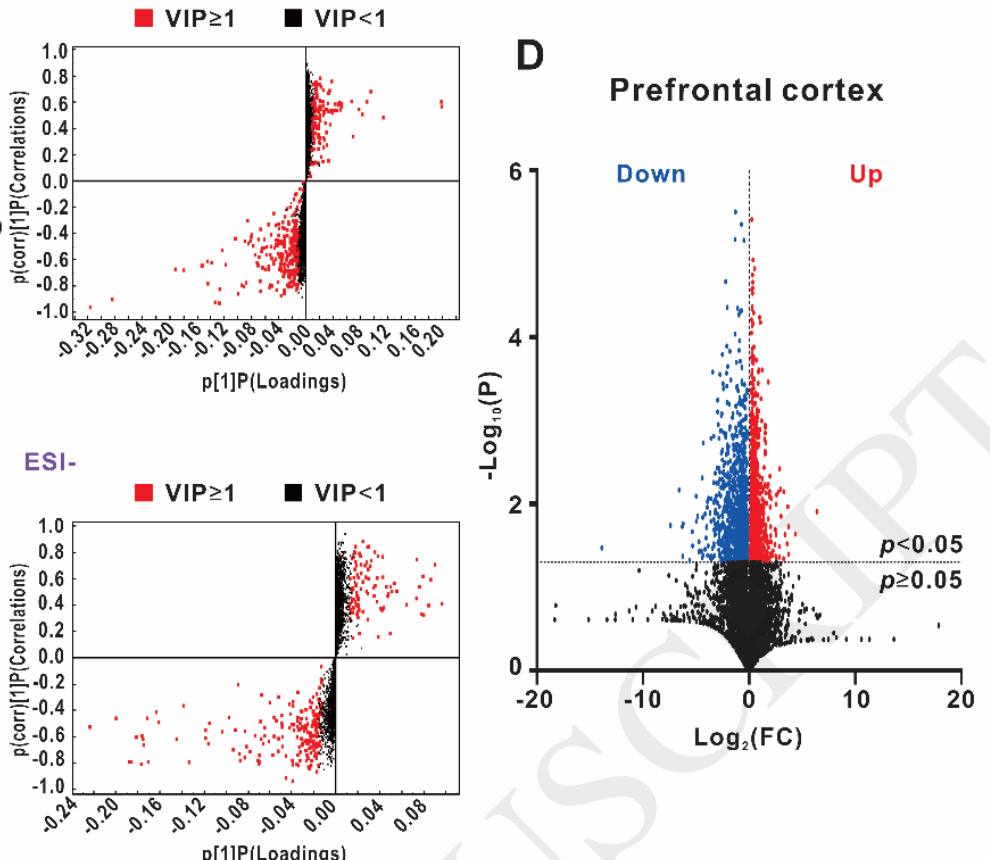

\section{E Striatum}

ESI+
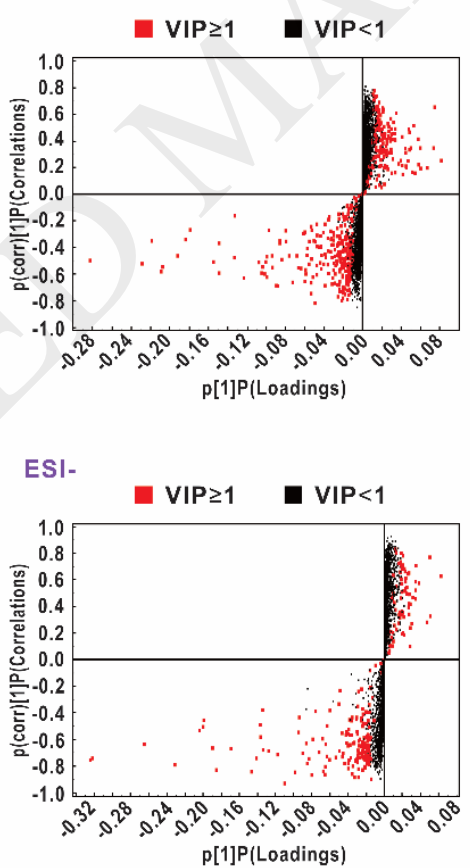

$\mathrm{p}[1] \mathrm{P}$ (Loadings)
$\mathbf{F}$

Striatum

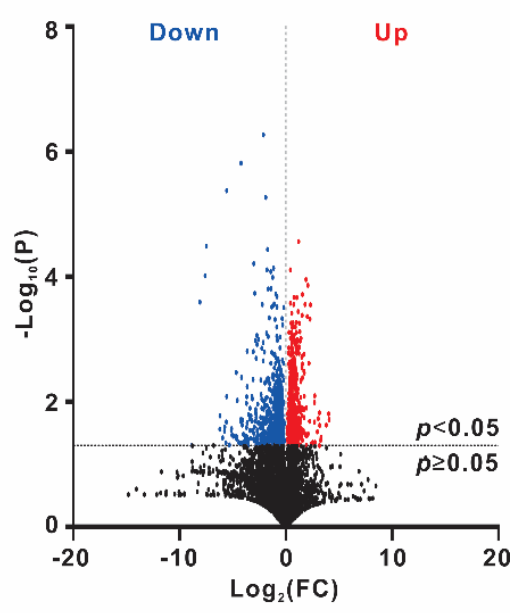




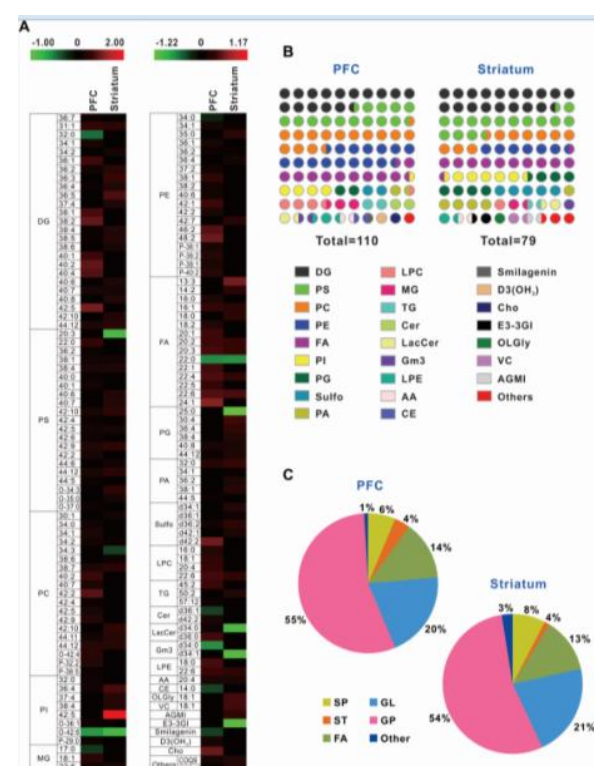


A Endoplasmic reticulum

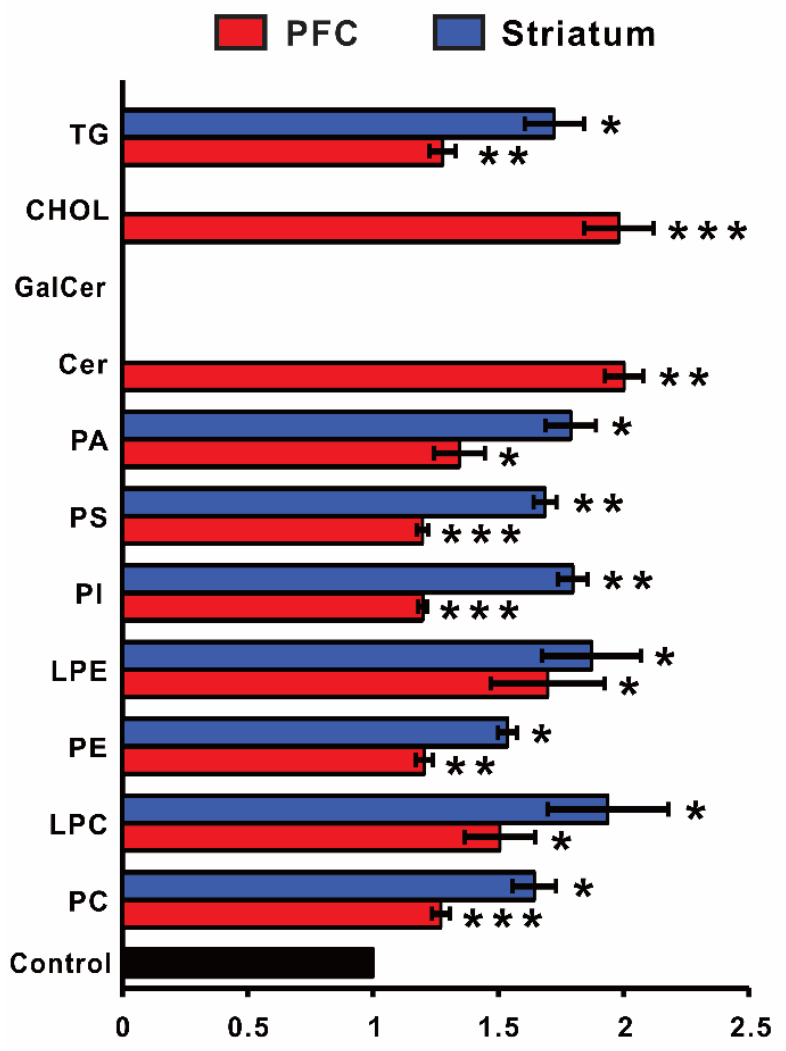

B

Mitochondria

$\square$ PFC $\square$ Striatum

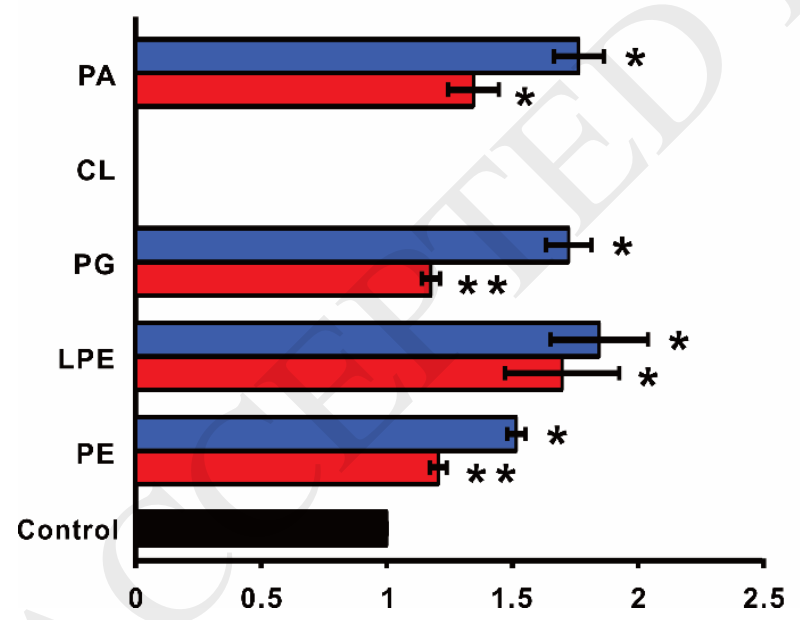

C

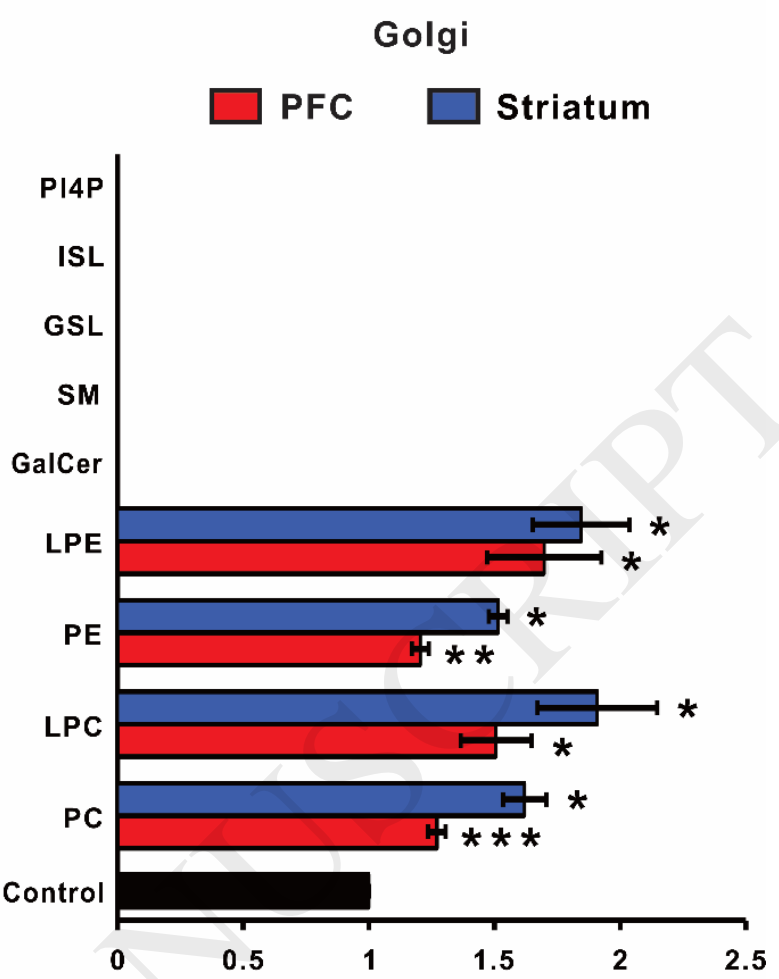

D

Plasma membrane

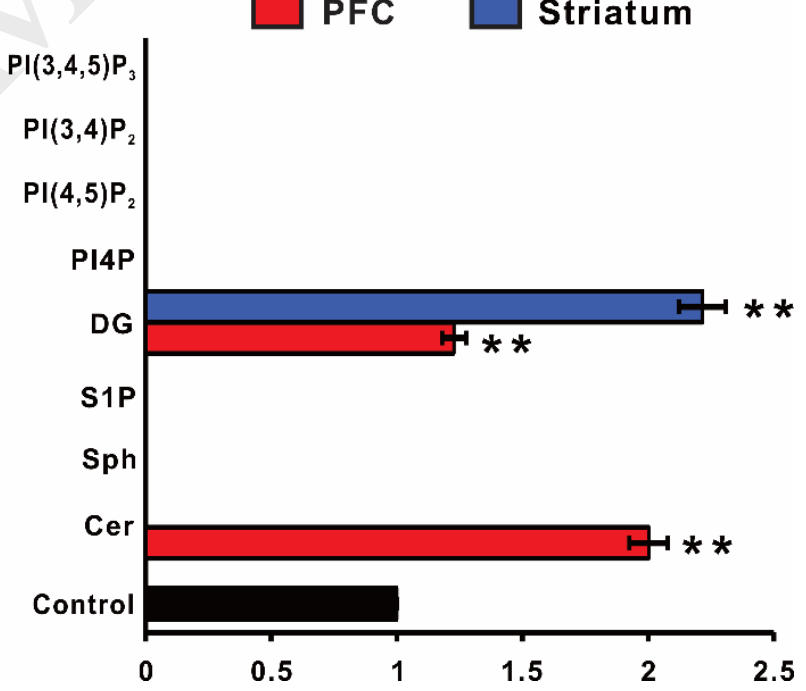


A

PFC

Saline $\square$ Alcohol

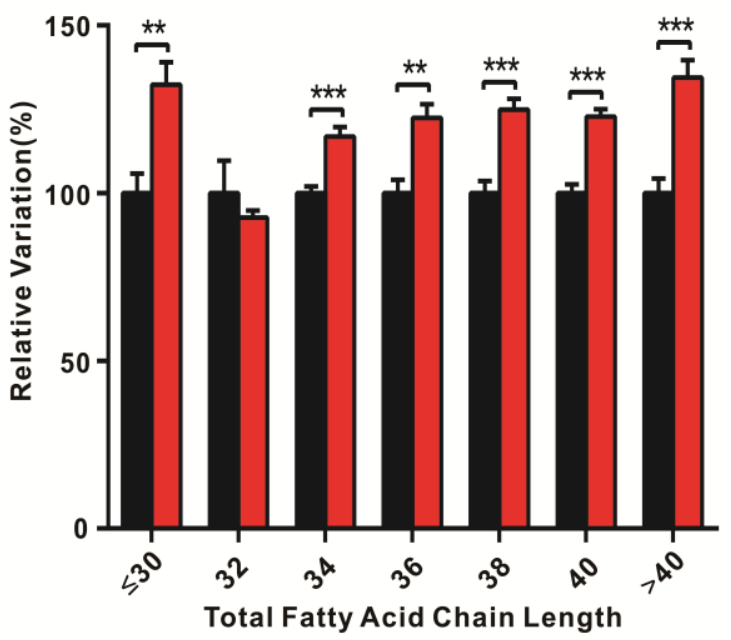

Striatum

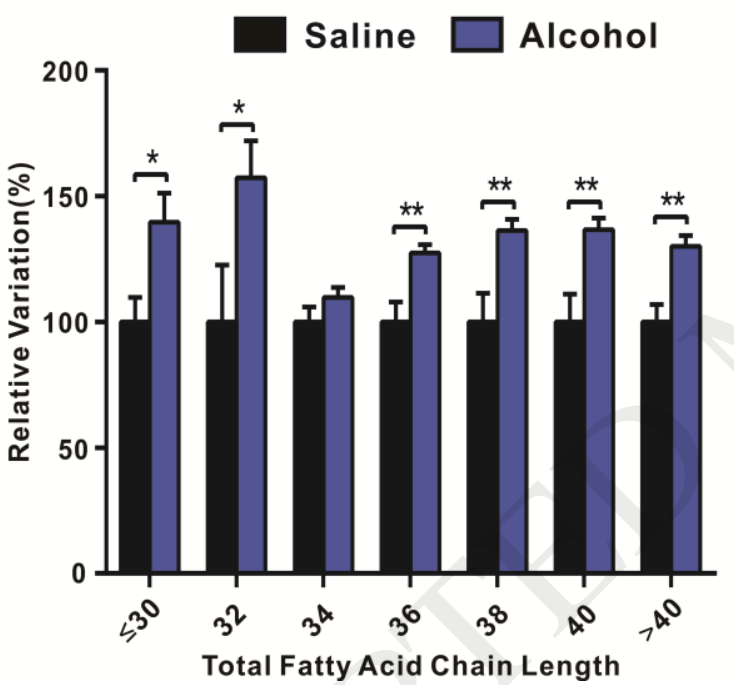

B PFC

Saline $\square$ Alcohol

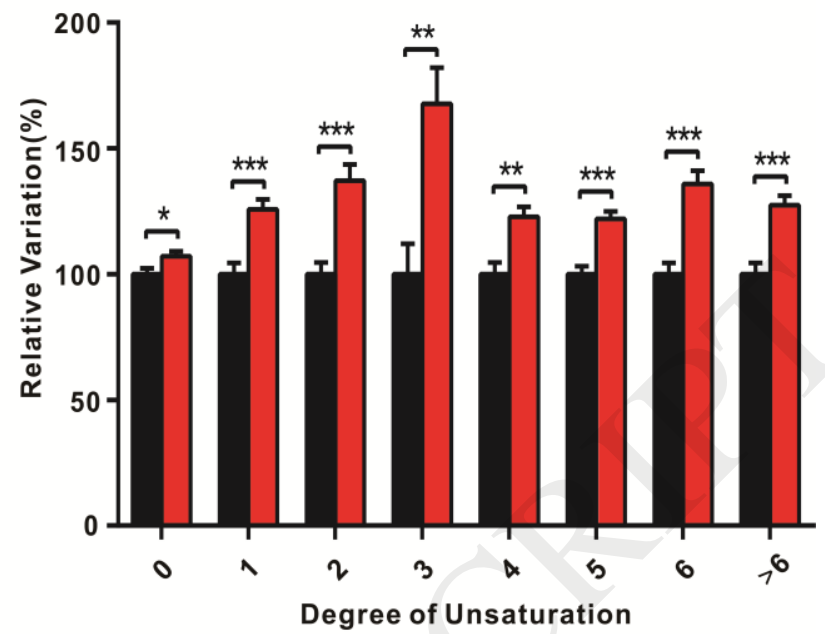

Striatum

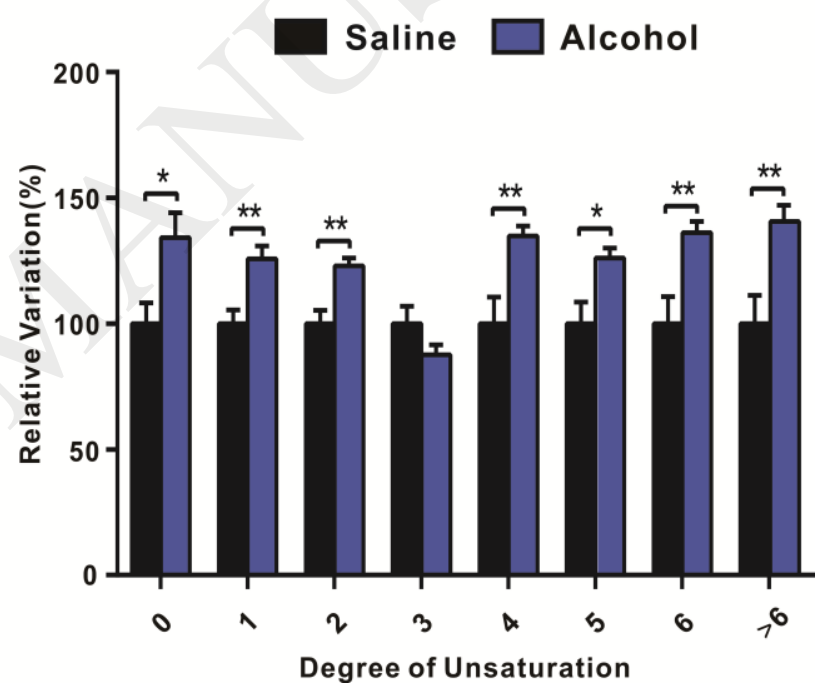




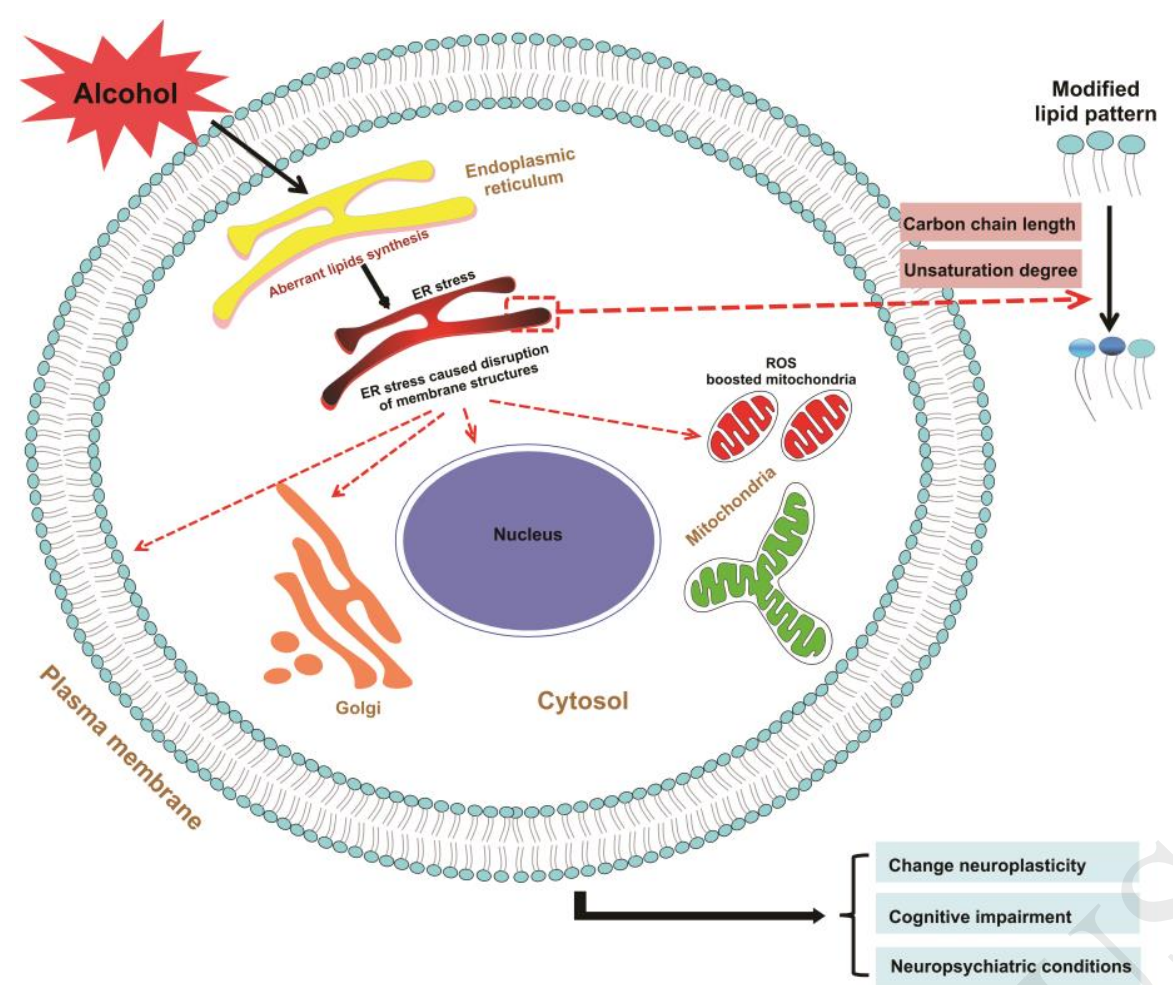

